\title{
AÇÃO DA DORAMECTINA INJETÁVEL SOBRE Haematobia irritans EM BOVINOS NATURALMENTE INFESTADOS: RESULTADOS DE OBSERVAÇÕES SIMULTÂNEAS NO BRASIL E ARGENTINA
}

\author{
ACTION OF INJECTABLE DORAMECTIN ON Haematobia irritans IN \\ CATTLE NATURALLY INFESTED: RESULTS OF SIMULTANEOUS \\ OBSERVATIONS IN BRAZIL AND ARGENTINA
}

\author{
João Ricardo Martins ${ }^{1}$ María MagdalenaVolpogni ${ }^{2}$ \\ Mirta Elda Castelli $^{3}$ Alberto Alejandro Guglielmone ${ }^{4}$
}

RESUMO

\begin{abstract}
Um teste de campo, realizado simultaneamente no Brasil (Eldorado do Sul, Rio Grande do Sul) e Argentina (Rafaela, Santa Fé), avaliou o comportamento da doramectina injetável sobre insetos adultos de Haematobia irritans. Em ambos os locais, utilizaram-se 40 animais $(20$ vacas e 20 novilhas), sendo que 20 foram tratados e 20 permaneceram como controle. Realizaram-se contagens de moscas nos dias 0, 1, 4, 7, 10, 14, 21, 28 e 35 pós-tratamento. Em comparação com o grupo controle, a eficácia observada no Brasil foi inferior a $50 \%$ no dia 1 , e 84,4, 72,6, 81,4, 74,2, 79,2, 50,7 e 64,7\% nos demais dias de observação enquanto na Argentina, foi de 97,3, 84,2, 95,9, 92,2, $93,6,63,3,73,8$ e 66,9\%, respectivamente. Em ambos os países, ocorreu uma contribuição dessa avermectina para a diminuição da população de insetos adultos de $\boldsymbol{H}$. irritans embora os resultados obtidos no Brasil tenham apresentado uma eficácia menor que os constatados na Argentina.
\end{abstract}

Palavras-chave: mosca-dos-chifres, Haematobia irritans, doramectina injetável, eficácia.

\section{SUMMARY}

A field test aiming an evaluation of the injectable doramectin on the horn-fly Haematobia irritans was carried out simultaneously in Brazil and Argentina. Both places, Eldorado do Sul, RS, Brazil and Rafaela, Santa Fé, Argentina, utilized a total number of forty adult animals being twenty treated and twenty controls. Flies were counted on days 0, 1, 4, 7, 10, 14, 21, 28 and 35 post-treatment (p.t.). By comparison with control group efficacy in Brazil was less than $50 \%$ in day +1 p.t., 84.4, 72.6, 81.4, 74.2, 79.2, 50.7 and $64.7 \%$ in the subsequent days while in Argentina was 97.3, 84.2, 95.9, 92.2, 93.6, 63.3, 73.8 and $66.9 \%$ respectively. A decrease on natural population of horn-fly was observed in both places indicating a contribution of this avermectin formulation in the control of this insect although results in Brazil showed less efficacy than in Argentina.

Key words: horn-fly, Haematobia irritans, injectable doramectin, field efficacy.

\section{INTRODUÇÃO}

A mosca-dos-chifres, Haematobia irritans, ingressou e se disseminou nos países do cone sul a partir de 1991 (LUZURIAGA et al., 1991, CARBALLO \& MARTINEZ, 1991) constituindo-se em mais um motivo de preocupação econômica para a bovinocultura. Os prejuízos econômicos decorrem da perturbação que demonstram os bovinos mais intensamente parasitados, fator que interfere no descanso e produtividade animal. Mais recentemente, consideráveis prejuízos na indústria do couro por ocasião da manipulação de peles de bovinos que sofreram altas infestações também são atribuídos a esses insetos (GUGLIELMONE et al., 1999b ).

\footnotetext{
${ }^{1}$ Médico Veterinário, MSc, FEPAGRO, Centro de Pesquisa Veterinária Desidério Finamor, Estrada do Conde, 6000, 92990-000, Eldorado do Sul, RS. E-mail: joaorsm@zaz.com.br. Autor para correspondência.

${ }^{2}$ Médico Veterinário, Atividade privada, Calle Dentesano 301, CP 2300, Rafaela (Santa Fé), Argentina.

${ }^{3}$ Bioquímica, INTA -Estación Experimental Agropecuária Rafaela, CP 2300, Rafaela (Santa Fé), Argentina

${ }^{4}$ Médico-Veterinário, PhD., INTA-Estación Experimental Agropecuária Rafaela, CP 2300 Rafaela (Santa Fé), Argentina.
} 
Os inseticidas continuam a ser o principal instrumento utilizado no controle da Haematobia irritans. Entretanto, o uso contínuo e muitas vezes inadequado dos químicos, tem favorecido o aparecimento e a expansão de problemas de resistência. Relatos na literatura evidenciam a ocorrência de resistência em diversos países, como os EUA, onde problemas de resistência a piretróides são detectados desde os anos 80 (KUNZ \& SCHMIDT, 1985), México (KUNZ et al., 1995; SANTAMARIA VARGAS $\boldsymbol{e t} \boldsymbol{a l} .$, 1995) e Argentina (TORRES et al.,1996; GUGLIELMONE et $\boldsymbol{a l}$., 1998) onde a diminuição de eficácia desses compostos tem resultado, paradoxalmente, em um incremento no uso desses inseticidas. Problemas de resistência a produtos organofosforados foram mencionados nos EUA (BARROS et al., 2001) e México (KUNZ et al., 1995), enquanto na Argentina e Brasil, em um recente levantamento (GUGLIELMONE et al., 2000), não foram detectadas populações com características de resistência a este grupo químico.

As avermectinas aplicadas sob a formulação injetável não estão indicadas para o tratamento de adultos de $\boldsymbol{H}$. irritans, ao contrário das formulações dorsais ("pour-on"), embora possuam notória ação no controle de larvas de insetos que utilizam substrato presente nas fezes bovinas (STRONG \& BROWN, 1987).

O uso desses endectocidas sistêmicos tem se intensificado nos últimos tempos especialmente para atender às demandas de manejo na bovinocultura, facilitadas pelo amplo espectro de ação desses químicos. $\mathrm{Na}$ intenção de se avaliar o comportamento de uma formulação injetável das avermectinas no controle da mosca-dos-chifres, optou-se pela doramectina, tendo em vista a sua disponibilidade e o tempo de existência nos mercados brasileiro e argentino.

\section{MATERIAL E MÉTODOS}

O presente trabalho foi realizado simultaneamente em Eldorado do Sul, RS, Brasil (latitude, 30.04S; longitude, 51.44W; altitude $47 \mathrm{~m}, 19,5^{\circ} \mathrm{C}$ e $1322 \mathrm{~mm}$ de precipitação média anual) e Rafaela, SF, Argentina (latitude, 31. 12S; longitude, $61.29 \mathrm{~W}$; altitude $99 \mathrm{~m}$; $19,2^{\circ} \mathrm{C}$ e $946 \mathrm{~mm}$ de temperatura e precipitação média anual, respectivamente). Em ambos os locais, foram utilizados 40 animais, raças cruzas de origem européias, sendo 20 vacas e 20 novilhas. Os dois grupos, tratados e controle, foram formados ao acaso, proporcionalmente ao número de vacas e novilhas. $\mathrm{O}$ produto utilizado foi a doramectina injetável (Dectomax $\left.{ }^{\circledR}\right)$, na dosagem de $200 \mu \mathrm{g} / \mathrm{kg}$, O início do tratamento ocorreu em 19 de fevereiro de 2001 sendo que as contagens de moscas, através de visualização direta dos animais no local onde se encontravam, foram realizadas nos dia $0,1,4,7,10,14,21,28$ e 35 , entre 08:30 e 10h, sempre pelo mesmo observador. Os bovinos permaneceram no mesmo potreiro onde foram realizadas as contagens, durante as cinco semanas do experimento, com uma distância aproximada de 600 metros entre o potreiro do grupo tratado e do controle. Para o cálculo da eficácia inseticida, foi utilizada a fórmula de HENDERSON \& TILTON (1955), comparando-se separadamente os grupos tratados e controle no Brasil e Argentina.

\section{RESULTADOS}

A tabela 1 mostra as médias dos resultados das contagens de moscas observados no Brasil e Argentina, até as 5 semanas pós-tratamento bem como os percentuais de eficácia obtidos. No Brasil, o melhor período de controle foi obtido entre os dias 4 e 21, sendo que a maior eficácia ocorreu no dia $4(84,4 \%)$. Na Argentina, este período correspondeu aos dias 1 e 14 pós-tratamento e maior eficácia ocorreu no dia 1 (97,3\%). Em quatro oportunidades as observações superaram 90\%, enquanto no Brasil, em nenhuma ocasião este percentual foi constatado.

\section{DISCUSSÃO}

ALVES-BRANCO et $\boldsymbol{a l}$. (1997) observaram uma eficácia na redução de moscas de $29,8 \%$ aos 14 dias, no terceiro ano de uso de doramectina injetável. Entretanto, doramectina na

Tabela 1 - Infestações médias por Haematobia irritans no grupo de bovinos tratados com doramectina injetável e grupo controle em experimentos simultâneos realizados no Brasil e Argentina e percentual de eficácia observado em cada país.

\begin{tabular}{lcccccc}
\hline & \multicolumn{3}{c}{ BRASIL } & \multicolumn{3}{c}{ ARGENTINA } \\
\cline { 2 - 7 } Dia & Tratado & Controle & \% eficácia & Tratado & Controle & \% eficácia \\
\hline 0 & 35,0 & 30,0 & na * & 90,5 & 98,0 & na* \\
1 & 27,0 & 44,4 & na * & 1,30 & 52,3 & 97,3 \\
4 & 12,0 & 65,5 & 84,4 & 13,1 & 89,6 & 84,2 \\
7 & 26,7 & 82,8 & 72,6 & 5,80 & 153,0 & 95,9 \\
10 & 11,4 & 52,5 & 81,4 & 6,00 & 83,5 & 92,2 \\
14 & 14,2 & 46,7 & 74,2 & 6,60 & 112,2 & 93,6 \\
21 & 13,7 & 56,4 & 79,2 & 68,3 & 201,5 & 63,3 \\
28 & 24,3 & 41,9 & 50,7 & 73,2 & 303,0 & 73,8 \\
35 & 30,9 & 74,4 & 64,7 & 93,1 & 304,5 & 66,9 \\
\hline
\end{tabular}

* não aplicável

** $\mathrm{P}>0,05$ Teste de Mann - Whitney 
formulação dorsal, apresentou uma eficácia acima de $92,8 \%$ pelo menos por 6 semanas após o tratamento (FARKAS et al., 2000). ANZIANI et al. (1999) observaram uma variação de eficácia de 66 (dia 21 pós-tratamento) a $81 \%$ (dia 7 pós-tratamento) nos 35 dias posteriores à aplicação de doramectina injetável a bovinos na Argentina. GUGLIELMONE et al. (1999a) observaram uma percentagem de redução da população de $\boldsymbol{H}$. irritans nos primeiros 14 dias pós-tratamento, a qual variou de 51 a mais de $73 \%$ em bovinos submetidos a tratamentos com abamectina injetável a cada 45 ou 63 dias, sugerindo que este composto pode auxiliar no controle das populações desse inseto, mantendo-as abaixo do limiar econômico.

A ação mosquicida inicial das avermectinas estaria baseada na mortalidade de moscas adultas que ingerem sangue com concentrações tóxicas (MILLER et al., 1986). Posteriormente, essa ação seria devido exclusivamente à inibição do ciclo pré-parasitário de $\boldsymbol{H}$. irritans no material fecal dos bovinos (FINCHER, 1992; DOHERTY et al., 1994; GUGLIELMONE et al., 1999a). Em termos gerais, a doramectina apresentou uma eficácia satisfatória para o controle da $\boldsymbol{H}$. irritans. Entretanto, notou-se diferenças nos valores de eficácia obtidos no Brasil e Argentina no presente estudo, a exemplo de outros anteriormente mencionados.

As causas dessas diferenças são difíceis de julgar. Alguns fatores de importância nesse sentido podem ser as diferentes condições ambientais para o desenvolvimento da $\boldsymbol{H}$. irritans (por exemplo, a precipitação observada no mês de fevereiro/2001 foi de 102,5mm em Eldorado do Sul, RS e de 19,2mm em Rafaela, SF); as diferentes pastagens das quais se alimentam os bovinos podem resultar em diferentes valores nutritivos da matéria fecal para os estádios pré-parasitários de $\boldsymbol{H}$. irritans (KUNZ, 1980) e, especialmente, as diferenças nas migrações de $\boldsymbol{H}$. irritans a partir das populações circundantes (MARLEY et al., 1991) podem afetar de forma diferencial a eficácia de um mesmo produto. Outros fatores como diferenças no metabolismo da droga, pelagem e tamanho dos animais podem contribuir para que os resultados de um mesmo tratamento não sejam idênticos.

As estratégias para o manejo da moscados-chifres devem incluir o uso de rotação de compostos com estruturas e modo de ação diferentes da dos piretróides, principal grupo inseticida atualmente utilizado para o seu controle, tendo em vista que pode ser esperado um aumento de populações resistentes a esses compostos em conseqüência desse uso contínuo (KUNZ et al.,
1995). Os organofosforados passam a ser a primeira opção química alternativa aos piretróides nos países do cone sul. Por outro lado, considerando esta assertiva, pode-se levar em conta que, com os resultados obtidos no presente trabalho, a doramectina também pode auxiliar no controle da mosca-dos-chifres, constituindo-se em uma alternativa para ser agregada às opções de compostos químicos, embora não seja recomendada, via injetável, como um produto específico para essa finalidade.

\section{REFERÊNCIAS BIBLIOGRÁFICAS}

ANZIANI, O.S., GUGLIELMONE, A.A. FLORES, S.G., $\boldsymbol{e}$ t al Evaluación de doramectina inyectable para el control de infestaciones naturales de Haematobia irritans (Diptera: Muscidae) en bovinos. Veterinaria Argentina, v. 16, p. 501-505, 1999.

ALVES-BRANCO, F.P.J., PINHEIRO, A.C., SAPPER, M.F.M. Atividade mosquicida da decametrina e doramectina em infestação natural de bovinos pela Haematobia irritans. Revista Brasileira de Parasitologia Veterinária, v.6. n.2, $\sup \ell .1$, p.43, 1997.

BARROS, A.T.M., OTTEA, J., SANSON, D., et al. Horn fly (Diptera:Muscidae) resistance to organophosphate insecticides. Veterinary Parasitology, v.96, p.243-256, 2001.

CARBAllo, M., MARTINEZ, M. Hallazgo de Haematobia irritans en Uruguay. Veterinária, v.27, p.20-21, 1991.

DOHERTY, W.M., STEWART, N.P., COBB, R.M., et al. In vitro comparison of the larvicidal activity of moxidectin and abamectin against Onthophagus gazella (F.) (Coloeptera: Scarabeidae) and Haematobia irritans exigua De Meijere (Diptera: Muscidae). Journal of the Australian Entomological Society, v.33, p.71-74, 1994.

FARKAS, R., GRÄFNER, G., HENDRICKX, M-O. Persistent efficacy of doramectin pour-on against Haematobia irritans in cattle. Veterinary Record, v.146, p.378-380, 2000.

FINCHER, G. Injectable ivermectin for cattle: effects on some dung - inhabiting insects. Environmental Entomology, v.21, p.871-876, 1992 .

GUGLIELMONE, A.A., KUNZ, S.E., VOLPOGNI, M.M., et al. Diagnóstico de poblaciones de la Haematobia irritans (Diptera:Muscidae) resistentes a la cipermetrina en Santa Fe, Argentina. Revista de Medicina Veterinaria, v.79, p.353356,1998 .

GUGLIELMONE, A.A.; VOLPOGNI, M. M.; ANZIANI, O.S., et al. Evaluation of injectable abamectin to control natural infestations of Haematobia irritans (Diptera:Muscidae) in cattle. Journal of Medical Entomology, v.36, n.3, p.325328, 1999a.

GUGLIELMONE, A.A., GIMENO, E., IDIART, J., $\boldsymbol{e}$ t al. Skin lesions and cattle hide damage from Haematobia irritans infestations in cattle. Medical and Veterinary Entomology, v.13, p.323-328, 1999 b. 
GUGLIELMONE, A.A., KUNZ, S.E., CASTELLI, M.E., et al. Susceptibilidad al diazinón de la Haematobia irritans (Diptera:Muscidae) de diferentes localidades argentinas y del sur de Brasil. Revista de Medicina Veterinaria, v.81, n.3, p.184-186, 2000.

HENDERSON, C.F., TILTON, E.W. Tests with acaricides against brown wheat mite. Journal of Economic Entomology, v.48, p.157-161, 1955.

KUNZ, S.E. Horn fly production as affected by seasonal changes in rangeland forage conditions.

The Southwestern Entomologist, v.5, p.80-83, 1980.

KUNZ, S.E., SCHIMIDT, C.D. The pyrethroid resistance problem in the horn fly. Journal of Agriculture and Entomology, v.2, p.358-363, 1985.

KUNZ, S.E., ESTRADA, M.O., SANCHEZ, H. F. Status of Haematobia irritans (Diptera:Muscidae) insecticide resistance in Northeastern Mexico. Journal of Medical Entomology, v.32, n.5, p.726-729, 1995.

LUZURIAGA, R.,EDDI, C., CARACOSTANTOGOLO, J., et al. Diagnóstico de parasitación com Haematobia irritans (L.) en bovinos de Misiones, Argentina. Revista Medicina Veterinaria, v.72, p.262-263, 1991.
MARLEY, S.E., LOCKWOOD, J.A., BYFORD, R.L., et al. Temporal, climatic, and physiological mediation of dispersal in the horn fly (Diptera: Muscidae). Environmental Entomology, v.20, p.1612-1618, 1991.

MILLER, J.A., OEHLER, D.D., SIEBENALER, A.J., et $\boldsymbol{a}$. Effect of ivermectin on survival and fecundity of horn flies and stable flies (Diptera: Muscidae). Journal of Economic Entomology, v.79, p.1564-1569, 1986.

SANTAMARIA VARGAS, M., ORTIZ ESTRADA, M., FRANCO BELLO, R., et al. Evaluación biológica de mosquicidas para el control de Haematobia irritans en Mexico y situación actual de la resistencia. In: SEMINÁRIO INTERNACIONAL DE PARASITOLOGIA, 3, 1995, Acapulco, México. Proceedings... INIFAP, 1995. p.1191239.

STRONG, L., BROWN, T.A. Avermectins in insect control and biology: a review. Bulletin of Entomological Research, v.77, p.357-389, 1987.

TORRES, P., BAIBI, A., SHEPPARD, D., et al. Resistencia de la mosca de los cuernos Haematobia irritans irritans ((L.1758) al Fenvalerato en la provincia de Corrientes, Argentina. Revista de Medicina Veterinária, v.77, p.136140,1996 